\title{
La formación de educadores en un mundo globalizado: Mirando hacia África
}

\author{
González Pérez, María Inmaculada \\ Universidad de la Laguna, Tenerife, España \\ migonpe@ull.edu.es
}

\author{
Astorgano Arias, Marta \\ Universidad de la Laguna, Tenerife, España \\ astorganoariasm@gmail.com
}

\section{Resumen}

La experiencia en materia de Cooperación Educativa Internacional (CEI) con África es muy reciente en la Facultad de Educación de la Universidad de La Laguna (ULL). Ésta se inició en 2005 con el Programa de Formación de Profesorado en los campamentos saharauis, mediante un convenio de colaboración firmado entre la Universidad de La Laguna y el Ministerio de Enseñanza y Educación de la República Árabe Saharaui Democrática. Destacó en esta iniciativa la implicación de los Departamentos de Didáctica e Investigación Educativa, de Psicología Evolutiva y de la Educación y Didácticas Específicas.

Ese mismo año tuvo lugar un proyecto de CEl entre Canarias y Mauritania titulado «A través de tus ojos: el agua» gracias a Médicos del Mundo, la Red Canaria de Escuelas Solidarias, la Consejería de Educación del Gobierno de Canarias y la Dirección General de Educación de Mauritania. Esta experiencia contó con la colaboración del Área de Teoría e Historia de la Educación. De su evaluación derivó en 2011 el proyecto titulado «Entre escuelas» que tuvo como objetivo principal el intercambio cultural.

Entre 2012 y 2015, destacaron los proyectos desarrollados con la Universidad de Cabo Verde (uniCV), donde la movilidad del alumnado y la publicación de materiales docentes cobraron especial relevancia. Estas iniciativas pudieron llevarse a cabo gracias al convenio firmado entre el Departamento de Historia y Filosofía de la Ciencia, la Educación y el Lenguaje de la ULL y el Departamento de Ciencias Sociales y Humanidades de la uniCV.

En septiembre de 2017 se firmó el convenio entre la Facultad de Educación y la Universidad de Tifariti en la República Árabe Saharaui Democrática, con el objeto de impulsar el intercambio de profesorado y alumnado. Todas estas experiencias han pretendido contribuir a la formación de pedagogos y docentes comprometidos, conscientes de necesidad de la solidaridad y responsabilidad internacional, capaces de transmitir estos principios en su labor profesional.

\section{Abstract}

The experience in the field of International Cooperation in Education (ICE) with Africa is very recent in the Faculty of Education of La Laguna University (ULL). This was initiated in 2009 through the Teacher Training Program in the Saharawi camps, through a collaboration agreement signed between the University of La Laguna and the Ministry of Teaching and Education of the Saharan Arab Democratic Republic. The involvement of the Departments of Didactics and Educational Research and Evolutionary Psychology and Education stands out in this initiative.

Later in 2011, an ICE project between the Canary Islands and Mauritania, entitled «Between Schools», was held thanks to Doctors of the World, the Canary Islands Network of Solidarity Schools, the Ministry of Education of the Government of the Canary Islands and the General Directorate of Education of Mauritania. This experience had the collaboration of the Area of Theory and History of Education. Its main objective was the intercultural exchange, having as a thematic axis: "Through your eyes: the water".

Between 2012 and 2015, the projects developed with the University of Cape Verde (uniCV) are especially relevant, being the mobility of students and the publication of teaching materials particularly important. These initiatives could be carried out thanks to the agreement signed between the Department of History and Philosophy of Science, Education and Language of the ULL and the Department of Social Sciences and Humanities of the uniCV.

In September 2017, the signing of the agreement between the Faculty of Education and the University of Tifariti in the Saharan Arab Democratic Republic took place, in order to promote the exchange of teachers and students.

All these experiences aim to contribute to the training of pedagogues and committed teachers, aware of the need for international solidarity and responsibility, capable of transmitting these principles in their professional work. 
Palabras clave: Cooperación Educativa Internacional, Formación docente, Formación pedagógica, Canarias, África.

Keywords: International Cooperation in Education, Teacher training, Pedagogical training, Canary Islands, Africa.

\section{INTRODUCCIÓN}

«La cooperación al desarrollo debe ser entendida como una relación entre países que persiguen un beneficio mutuo, con unos fines concretos: consolidación democrática, desarrollo humano, social y económico sostenible, lucha contra la pobreza, protección del medio ambiente, entre otros. Todo ello planteado en términos de corresponsabilidad entre las contrapartes» (García de la Torre, 2011:89).

Atendiendo a esta definición, en el ámbito universitario tienen lugar numerosas experiencias que se manifiestan principalmente en los terrenos de la investigación, la asesoría técnica y la formación de grado o postgrado.

Las universidades españolas, especialmente durante este siglo, han dado un notable impulso a la cooperación educativa al desarrollo en su empeño de internacionalización. La mayoría tiene un departamento de cooperación al desarrollo y una red de contactos que les permite poner en marcha programas de cooperación o de codesarrollo (Demamesse, 2006:14).

No ajena a este esfuerzo, la Universidad de La Laguna y más concretamente su Facultad de Educación, han puesto en marcha iniciativas en África con el objeto de aprovechar las potencialidades que ofrece la proximidad geográfica de las Islas Canarias con este continente. A continuación se detallan dichos esfuerzos que contribuyen a un desarrollo humano sostenible, justo y equitativo.

\section{PROYECTO A TRAVÉS DE TUS OJOS: EL AGUA}

Entre 2005 y 2007 tuvo lugar un proyecto de CEI entre Canarias y Mauritania titulado «A través de tus ojos: el agua» gracias a Médicos del Mundo, la Red Canaria de Escuelas Solidarias, la Consejería de Educación del Gobierno de Canarias y la Dirección General de Educación de Mauritania.

Esta experiencia, coordinada por la especialista en Educación para el Desarrollo, Ana Esther Barrios Sánchez, contó en su última fase con la colaboración del Área de Teoría e Historia de la Educación del Departamento de Historia y Filosofía de la Ciencia, la Educación y el Lenguaje de la Universidad de La Laguna. Concretamente participaron las profesoras Mercedes García de la Torre Gómez e Inmaculada González Pérez. Las actividades realizadas implicaron desplazarse a Mauritania ya que tenían como objetivos:

- «Conocer el entorno, la forma de vida y las características culturales de Nouadhibou.

- Desarrollar un estudio comparativo entre los sistemas educativos de Canarias y Mauritania para el fomento del enriquecimiento mutuo.

- Establecer las bases para el proyecto de «Cooperación Educativa para el Desarrollo Nouadhibou - Canarias» (RECS, 2007:7).

Entre todas las acciones implementadas, es necesario destacar los seminarios conjuntos con el profesorado mauritano. El contacto con los docentes permitió conocer en profundidad el funcionamiento del sistema educativo del país y la realidad educativa de Nouadhibou. «En este sentido, no sólo fueron importantes las necesidades detectadas, sino también las potencialidades, especialmente los recursos humanos existentes. El profesorado, a pesar de las limitaciones materiales, pone un enorme empeño en lograr el aprendizaje de su alumnado. Así mismo, destacamos su enorme concreción a la hora de establecer las prioridades en materia de cooperación educativa, centrándose en los intereses del alumnado» (RCES, 2007:8). 
La evaluación de este proyecto propició el diseño de un nuevo proyecto de CEI titulado «Entre Escuelas» que se presenta en el apartado 3.

\section{PROGRAMA DE FORMACIÓN DEL PROFESORADO EN LOS CAMPAMENTOS SAHARAUIS}

Este programa titulado «Formación del Profesorado en los Campamentos Saharauis» se llevó a cabo en 2009 entre la Facultad de Educación de la Universidad de La Laguna (ULL) y el Ministerio de Enseñanza y Educación de la República Árabe Saharaui Democrática (RADS). Para ello fue imprescindible la implicación de los Departamentos de Didáctica e Investigación Educativa, de Psicología Evolutiva y de la Educación y de Didácticas Específicas.

Sus principales objetivos fueron:

— «Propiciar la participación del personal académico de cada institución en todos los eventos científicos y culturales organizados por la otra.

- Elaborar programas y proyectos de cooperación, en los que se especificarán las obligaciones de cada una de las partes en la ejecución de los mismos. Estos programas y proyectos serán objeto de acuerdos complementarios o de ejecución, conforme a la normativa de cada institución.

- Desarrollar el intercambio de bibliografía, publicaciones, material docente, exposiciones o cualquier otra experiencia artística, de interés para ambas instituciones.

- Facilitar la aceptación preferente de profesores y alumnos de la otra institución que deseen continuar o completar los estudios impartidos por la propia, con especial mención a los programas de postgrado y doctorado» (De la Guardia y Gobante, 2009 a).

Dentro de las prioridades establecidas por Ministerio de Enseñanza y Educación de la República Árabe Saharaui Democrática, desde la Facultad de Educación se realizaron una serie de actividades que consistieron en la impartición de varios Seminarios de Formación a cargo de su profesorado dirigido al profesorado saharaui, así como en la realización de prácticas por parte de 38 alumnos y alumnas de la Facultad matriculados en sus diferentes titulaciones: Pedagogía, Maestro en Educación Infantil, Maestro en Educación Primaria, Lengua Extranjera, Educación Física y Educación Musical y Psicopedagogía. Las prácticas del alumnado contaron con el apoyo financiero de la Fundación Bancaja (de la Guardia y Gobante, 2009 b).

Este programa, bajo la responsabilidad de Rosa de la Guardia Romero y José María Gobante Ollero, se concretó en los siguientes proyectos:

— Instalación y dotación de materiales pedagógicos, bajo la responsabilidad de Rosa de la Guardia Romero.

- Formación en Psicomotricidad, a cargo del profesor Miguel Llorca Llinares.

- Organización y puesta en marcha del centro de profesores, siendo la persona responsable el profesor José M. ${ }^{a}$ Gobante Ollero.

- Prácticas de animación a la lectura, realizadas por la cuentacuentos Laura Escuela Magdaleno.

— Formación en didáctica de la enseñanza de la segunda lengua (español), impartida por profesor José Luis Vera Bautista.

— Formación en didáctica de las matemáticas activa, impartida por las maestras de matemáticas Victoria Soco y Luz M. ${ }^{a}$ Viera.

- Intercambio de experiencias con instituciones educativas canarias,

- Actividades lúdicas y culturales realizadas por el grupo de payasos CLOWNBARET (de la Guardia y Gobante, 2009:1-4). 


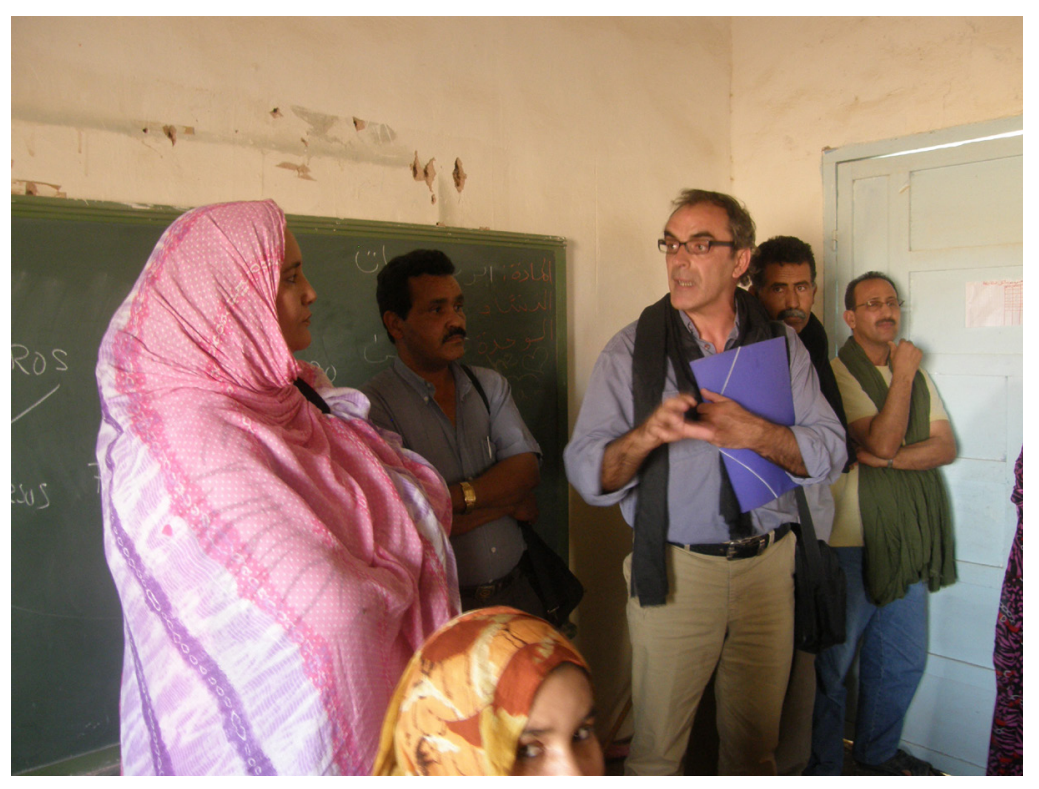

Profesorado de la ULL en los campamentos saharauis (José M. ${ }^{a}$ Gobante).

\section{PROYECTO ENTRE ESCUELAS}

El proyecto titulado «Entre Escuelas», diseñado en 2011 entre Nouadhibou y Canarias, tuvo como objetivo consolidar un marco de cooperación educativa internacional directo, autónomo y sostenido en el tiempo entre escuelas de ambos territorios. Fue realizado por Médicos del Mundo y la Red Canaria de Escuelas Solidarias con el apoyo del Área de Teoría e Historia de la Educación del Departamento de Historia y Filosofía de la Ciencia, la Educación y el Lenguaje de la Universidad de La Laguna. Concretamente participaron en su elaboración las profesoras Mercedes García de la Torre Gómez e Inmaculada González Pérez.

Bajo la coordinación de Damián Marrero Real, su estrategia fundamental residía en el establecimiento de redes educativas con relaciones directas y estrechas entre las escuelas y las instituciones que apuestan por la solidaridad, la interculturalidad y por la cooperación educativa al desarrollo para mejorar los sistemas de educación de ambas regiones.

Culminaba esta cooperación con la publicación de una «Guía para la Cooperación Internacional entre Escuelas», útil para el profesorado, siendo «un medio para apoyar la educación pública, que asuma retos a largo plazo; un instrumento de formación que facilite la comprensión mutua; una oportunidad de acercamiento a otras realidades mediante el intercambio de conocimientos y experiencias; un canal de comunicación e intercambio cultural» (RCES y Médicos del Mundo, 2011:11).

Este proyecto se vio truncado por el golpe militar que tuvo lugar en 2008 en Mauritania.

\section{REDES EN EL FORTALECIMIENTO INSTITUCIONAL ENTRE LAS UNIVERSIDADES DE CABO VERDE Y CANARIAS}

En 2012, la Agencia Española de Cooperación Internacional al Desarrollo financió el diseño del proyecto titulado «Redes para el fortalecimiento institucional entre las universidades de Cabo Verde y Canarias», dirigido a promover los estudios para el desarrollo para la formación de especialistas en contenidos relacionados con la educación y la cooperación para el desarrollo en la Universidad de Cabo Verde y la Universidad de La Laguna, concretamente entre la Facultad de Educación y el Departamento de Ciencias Sociales y Humanas- Curso de Ciencias de la Educación. Ambos organismos son los responsables de la formación de pedagogos y maestros.

"Las actividades que se realizaron fueron concebidas a medio y largo plazo, con vocación de continuidad y de beneficio mutuo, orientadas a los intereses y necesidades de los países y las universidades; respetuosas con los contextos sociales, políticos, culturales, medioambientales de los países y planteadas conforme a los principios de la cooperación y la educación para el desarrollo» (González-Pérez y García de Torre, 2012:3). 
Esta iniciativa fue promovida e implementada por las profesoras Mercedes García de la Torre Gómez e Inmaculada González Pérez del Área de Teoría e Historia de la Educación del Departamento de Historia y Filosofía de la Ciencia, la Educación y el Lenguaje. Su objetivo general era establecer un sistema de cooperación educativa al desarrollo y una red de comunicación directa y estrecha, sostenida en el tiempo entre la Universidad de Cabo Verde y la Universidad de La Laguna, con el propósito de fortalecer los sistemas de formación e investigación e intercambio en materia de educación y cooperación para el desarrollo. Y entre sus objetivos específicos destacaban los siguientes:

- Crear de una red interuniversitaria bilateral y multilateral.

- Mejorar la movilidad bidireccional en la formación inicial y continua.

- Diseñar una guía de cooperación educativa para el desarrollo entre universidades.

- Celebrar un Taller Internacional sobre redes para el fortalecimiento.

Los resultados más destacado de este proyecto fueron: 1) la consolidación de un grupo de investigación integrado por miembros de diversos países; 2) la disponibilidad de recursos para la formulación de proyectos; y 3 ) la formulación de un proyecto de cooperación educativa realizado conjuntamente entre el profesorado de ambas universidades. Este proyecto se presenta a continuación.

\section{REDES PARA LA FORMACIÓN Y LA INVESTIGACIÓN EN COOPERACIÓN Y EDUCACIÓN PARA EL DESARROLLO ENTRE LAS UNIVERSIDADES DE CABO VERDE Y LA LAGUNA}

El proyecto «Redes para la Formación y la Investigación en Cooperación y Educación para el Desarrollo entre las Universidades de Cabo Verde y La Laguna» (FICED) formuló e implementó, desde 2012 hasta 2014, acciones que contribuían al ejercicio horizontal de la cooperación educativa para el desarrollo (CEI) y la educación para el desarrollo (EpD). Fue financiado por el Fondo Europeo de Desarrollo Regional e implementado por las profesoras Mercedes García de la Torre Gómez, Margarita Rodríguez Rodríguez e Inmaculada González Pérez del Departamento de Historia y Filosofía de la Ciencia, la Educación y el Lenguaje.

Su objetivo fundamental fue el desarrollo y la consolidación de redes estables de cooperación educativa al desarrollo en el campo de la investigación y la docencia, el asesoramiento mutuo y la movilidad, además de la sensibilización de la comunidad universitaria hacia la solidaridad internacional, la desigualdad y la inclusión social.

En el ámbito de la formación, las actividades que se propusieron trataban de formar profesionales de la educación en Grado, Posgrado y otros niveles de especialización que requieren los proyectos de cooperación y educación al desarrollo. Para ello se propuso introducir en los programas de asignaturas o materias de las nuevas titulaciones contenidos sobre el desarrollo, la cooperación y la educación para el desarrollo. También pretendió mejorar el intercambio de estudiantes y profesores para un mayor conocimiento de los sistemas educativos (especialmente los universitarios) aquí y allí, mediante la realización de prácticas externas, el estudio de asignaturas ofertadas por las universidades implicadas y el intercambio de profesorado.

Las actividades de investigación consistieron en «la creación de redes universitarias para la construcción de conocimientos conjuntos, tratando de pasar de una lógica de transferencia del conocimiento, a una lógica de construcción conjunta del conocimiento, en campos como el desarrollo y la cooperación, lo que nos llevaría a realizar estudios de calidad y de impacto sobre las acciones de cooperación» (González-Pérez, García de la Torre y Rodrígues, 2013:109).

Otro aspecto importante de este proyecto fue la participación conjunta en programas de cooperación educativa al desarrollo; en la formulación, desarrollo, seguimiento y evaluación de acciones y proyectos de desarrollo, creando espacios de reflexión en las dos universidades para favorecer las interrelaciones culturales.

De todos los resultados alcanzados destacan las prácticas externas realizadas por el alumnado canario en Cabo Verde y los recursos disponibles para todo el profesorado y alumnado en materia de CEI:

Guía de Cooperación Educativa Internacional y Educación para el Desarrollo elaborada por profesorado canario y caboverdiano. Este material docente permite una planificación de la cooperación regio- 
nal de mejor calidad; sirviendo para orientar las acciones y programas en el marco de la cooperación educativa y la educación para el desarrollo en Cabo Verde y Canarias.

- Cooperación Educativa Internacional, píldora educativa en español y video tutorial en portugués de gran utilidad para profesorado y alumnado universitario.

- Educación para el Desarrollo, píldora educativa en español y video tutorial en portugués también de gran utilidad para profesorado y alumnado universitario.

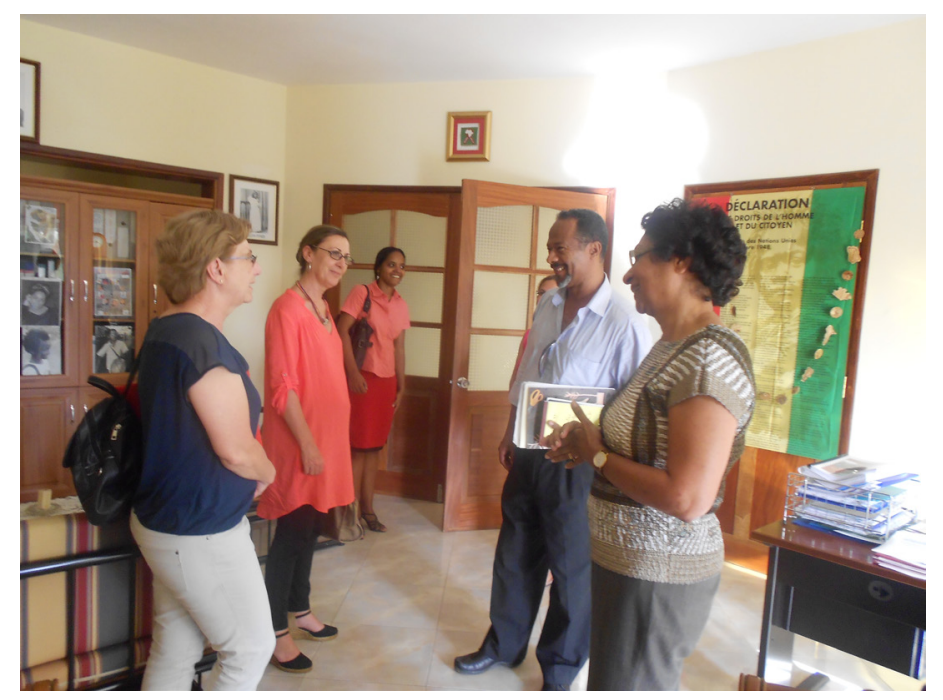

Profesorado de la ULL en Cabo Verde (Mercedes García de la Torre y Margarita Rodríguez).

\section{CONVENIO DE COOPERACIÓN CON LA UNIVERSIDAD DE TIFARITI}

Si la primera actividad de cooperación desarrollada por la Facultad de Educación de la Universidad de La Laguna se desarrolló con la RADS, también la última fue con este Estado mediante la firma en 2017 del acuerdo entre ésta y la Universidad de Tifariti para el desarrollo del proyecto titulado: Cooperación al desarrollo, educación, redes-Tics y patrimonio cultural.

El objetivo general de este convenio promovido por las profesoras Carmen Ascanio Sánchez e Inmaculada González Pérez es colaborar en el desarrollo de capacidades en relación con la docencia, la gestión y la investigación, trabajando conjuntamente en la cooperación y el intercambio de carácter académico. Sus objetivos específicos son:

— «Asesorar, intercambiar y ofrecer apoyo recíproco en las líneas de trabajo conjunto, con el foco de atención en el desarrollo de capacidades y redes.

- Difundir experiencias docentes, de gestión e investigación.

- Impartir seminarios, cursos y talleres en ambas universidades, afianzando las redes docentes y la difusión del conocimiento.

- Participar en proyectos docentes conjuntos, gestionando estancias para estudiantes, así como tutorizaciones conjuntas.

- Incentivar publicaciones conjuntas tanto de tipo monográfico como comparativo.

- Promover las estancias de profesorado/ PAS, para favorecer el intercambio de recursos humanos entre ambas instituciones.

- Diseñar proyectos de investigación conjuntos en las materias objeto del proyecto» (Ascanio y González-Pérez, 2017: 2).

Las posibilidades que se abren con este convenio con ilimitadas. Veremos la capacidad del profesorado y alumnado canario y saharaui para desarrollar acciones solidarias que permitan cubrir las necesidades de ambos pueblos. 


\section{CONCLUSIONES}

Los proyectos que han tenido lugar durante más de una década en la Facultad de Educación de la ULL con África hacen que ésta disponga en la actualidad de una valiosa experiencia en materia de solidaridad y cooperación educativa internacional.

Se ha iniciado un camino de enriquecimiento mutuo entre la universidad tinerfeña y africanas, siendo necesario profundizar en los logros y desaciertos con el objeto de mejorar y seguir avanzando en este imprescindible camino educativo.

Aún quedan mucho por andar. Quedan ámbitos que abarcar: la educación postobligatoria, la educación de adultos, la educación no formal... Quedan actores con los que trabajar: asociaciones de alumnado, asociaciones de madres y padres, organizaciones no gubernamentales... Quedan territorios que explorar: Senegal, Guinea, Mozambique...

Y quedan temáticas en las que profundizar... Respecto a esta última cuestión, atendiendo al elevado número de mujeres que cursan estudios relacionados con la educación tanto en Canarias como en África y la persistencia de las desigualdades en ambos lugares del planeta, quizás la defensa del derecho humano referente la igualdad de género pudiera ser priorizado de cara a un futuro próximo.

\section{REFERENCIAS BIBLIOGRÁFICAS}

Convenio específico de colaboración entre la Facultad de Educación de la Universidad de La Laguna (España) y la Universidad de Tifaritti de la República Árabe Saharaui (2017).

De la Guardia Romero, R. y Gobante Ollero, J. M. (2009 a). Proyecto del Programa de formación del profesorado en los campamentos saharauis - República Árabe Saharaui Democrática (RADS). (sin publicar).

De la Guardia Romero, R. y Gobante Ollero, J. M. (2009 b). Programa de formación del profesorado en los campamentos saharauis - República Árabe Saharaui Democrática (RADS). Nota de prensa Gabinete de Comunicación de la Universidad de La Laguna.

Demanesse, M. (2006). Educación formación y codesarrollo desde España, febrero. En (www.codesarrollo-cideal.org), consultado el 15 de noviembre de 2009.

García de la Torre Gómez, M. (2003). La Cooperación educativa para el Desarrollo, píldora educativa disponible en: http:// ullmedia.udv.ull.es/watch_video.php?v=URWX904NO34R.

García de la Torre Gómez, M. (2003). Cooperação Educativa para el Desenvolvimiento, vídeo tutorial disponible en: http:// ullmedia.udv.ull.es/watch_video.php?v= URWX904NO34R.

García de la Torre Gómez, M. y González Pérez, I (2011). Las universidades ante el reto de la Educación para el Codesarrollo. En Revista Española de Educación Comparada, Cooperación al Desarrollo en Educación: Alianzas con Educación Comparada desde la Perspectiva del Desarrollo, N. ${ }^{\circ} 17$, Madrid, UNED/SEEC, pp. 87-110.

González-Pérez, I., García de la Torre Gómez, M. y Rodrígues Spínola, C. (2016). Buscando nuevos caminos para la cooperación internacional interuniversitaria entre Cabo Verde y Canarias. En Valle, J.M. y Núñez, J.A. (coordinadores). Educación, supranacionalidad y ciudadanía. España: Fundación Santillana, pp. 103-115.

González-Pérez, I., García de la Torre Gómez, M. y Rodrígues Spínola, C. (coord.) (2013). Guía de Cooperación educativa internacional y educación para el desarrollo. Madrid: Biblioteca Nueva.

González-Pérez, I. (2013). Educación para el Desarrollo (EpD), píldora educativa disponible en http://ullmedia.udv.ull.es/ watch_video.php?v=B3AK3BK77619.

González-Pérez, I. (2013): Eduçao paea o desenvolvimento (EpD), video tutorial disponible en http://ullmedia.udv.ull.es/ watch_video.php?v=R62S8OG8MOY4.

González-Pérez, I. y García de la Torre Gómez, M. (2012). Proyecto Redes para el fortalecimiento institucional entre las universidades de Cabo Verde y Canarias (sin publicar).

Médicos del Mundo y RCES (2007). Proyecto A través de tus ojos: el agua (sin publicar). 
RCES (2007). Memorando del viaje del profesorado de la RCES a NOUADHIBOU en el marco del Proyecto A través de tus ojos: el agua (sin publicar).

RCES y Médicos del Mundo (2011). Proyecto Entre escuelas (sin publicar). 